www.jmscr.igmpublication.org

Index Copernicus Value: 79.54

ISSN (e)-2347-176x ISSN (p) 2455-0450

crossref DOI: https://dx.doi.org/10.18535/jmscr/v7i4.165

Journal Of Medical Science And Clinical Research

IGM Publication

An Official Publication of IGM Publication

\title{
Second hand smoke exposure in pregnancy and the risk of still birth - a case control study in a tertiary care centre in Kerala
}

\author{
Authors \\ Nirmala.C ${ }^{1^{*}}$, Raji.S ${ }^{2}$ K.T Shenoy ${ }^{3}$ \\ ${ }^{1}$ Department of Obstetrics and Gynecology, SAT Hospital, Govt. Medical College, Trivandrum \\ ${ }^{2}$ Junior Consultant, Credence Hospital, Ulloor, Trivandrum \\ ${ }^{3}$ Department of Gastroenterology, Sree Gokulam Medical College and Research Foundation, Trivandrum, \\ Kerala, India \\ *Corresponding Author \\ Dr C. Nirmala \\ Department of Obstetrics and Gynecology, SAT Hospital, Govt. Medical College, Trivandrum
}

\begin{abstract}
Introduction: Second hand smoke exposure also known as passive smoking is a global health problem with profound impact on the expectant mother and her unborn baby. Still birth, the absence of life at birth, is a major obstetric problem. Ending preventable stillbirth is a global priority and hence assessing the causes is important. Association between second hand smoke exposure in pregnancy and stillbirth is hypothesized. But little is known about the role of second hand smoke and antepartum stillbirths in Kerala where the prevalence smoking among men is high.
\end{abstract}

Aims and Objectives: Primary Objective was to find the association between passive smoke exposure and the risk of still birth.

Methodology: This is a case control study conducted in the department of Obstetrics \& Gynaecology, Govt. Medical College, Trivandrum, a tertiary care teaching hospital. 64 cases of sonologically confirmed antepartum still birth was compared with 128 live births (case control ratio 1: 2). Exposure to passive smoking was assessed by structured questionnaire and self report of passive smoking was quantified by estimating salivary continine which is a byproduct of nicotine using ELISA test. Salivary cotinine assay has a high sensitivity and specificity and is a non invasive test.

Results: The risk of antepartum stillbirth was 7 times higher among prenatal women exposed to second hand smoke as compared to those without exposure (OR-7.97, 95\% CI-4.01 to 15.84). There is a linear relationship between the duration and extent of exposure. Other risk factors are age > 30 years, lower socio economic status, increased BMI, gestational hypertension .On logistic regression analysis for confounding variables, second hand smoke exposure was found to have an independent association with stillbirth (P value < 0.001).

Conclusion: Based on our study we conclude that there is an association between prenatal second hand smoke exposure and risk of stillbirth. This study is first of its kind in a state of Kerala, with a high rate of smoking among men. The results of this study shows that there is an urgent need for smoking cessation thereby avoiding passive smoke exposure to women and their unborn babies.

Keywords: second hand smoke, stillbirth, intrauterine death. 


\section{Introduction}

Still birth, the absence of life at birth, is a major obstetric problem. Even though globally antepartum and intrapartum care has improved, stillbirth remains a major challenge and this is a largely unresolved. Still birth commonly known as intrauterine death can happen either during antepartum period or during the process of labour. Stillbirth (according to $\mathrm{WHO})^{1}$ is defined as the death of the fetus in uterus before birth at or after 22 weeks of gestation or when the fetus is 500 gram, if the gestational age is not known. ${ }^{1}$ Causes of still births are multifactorial. Exposure of nonsmoking mother to second hand smoke (SHS) is proposed as a risk factor for preventable stillbirth.

Exposure to secondhand smoke (SHS), also known as passive smoking or environmental tobacco smoke (ETS) is considered as serious threats to the health of pregnant women, infants, and children around the world. ${ }^{2,3}$ In many highincome countries, tobacco use is a leading preventable cause of poor pregnancy and infant outcomes, such as low birth weight, preterm delivery, placental abruption, and sudden infant death syndrome ${ }^{4}$

Secondhand tobacco smoke (SHS), also referred to as passive smoking or environmental tobacco smoke, is a complex aerosol made up of carcinogenic, mutagenic, and toxic compounds. There are two types of SHS, mainstream smoke and side stream smoke. ${ }^{5}$ The effect due to chemical exposure from second-hand smoke is similar to the exposure of the smoker, but the pattern and amounts of exposure vary and are different from that of the smoker. Secondhand smoke (SHS) is composed of side stream smoke emitted from the smouldering tip of a cigarette $(80 \%$ to $90 \%)$ and exhaled mainstream smoke (10\% to $20 \%)$. Mainstream smoke is the tobacco smoke that is created when a smoker inhales the burning tobacco and then exhales. Side stream smoke is the smoke components that are released from the end of the smoldering tobacco product. Various toxic gasses, such as acrolein, benzene, carbon monoxide, and formaldehyde are found in side stream smoke, many of which are carcinogenic and cause non-cancer health related effects. Over five million deaths are attributable to smoking worldwide, and over 600,000 are linked to SHS exposure yearly. According to a 2006 US Surgeon General Report, "The scientific evidence indicates that there is no risk-free level of exposure to secondhand smoke". ${ }^{3}$ The detrimental effects of secondhand tobacco smoke exposure begins in utero as the placenta offers no barrier SHS exposure. The period of intrauterine growth and development is one of the most vulnerable periods in the human life cycle.

Ending preventable stillbirth in every country by 2030 is one of the targets of sustainable development goals (SDG). ${ }^{6,7}$. Second hand smoke exposure is being recognized globally as a threat to intrauterine life. But the impact is less clear than the impact of direct smoking. Most of the evidence on SHS exposure during pregnancy is from developed countries. In many developing countries there are not many well conducted studies to assess the effect on the foetus. In most of the South East Asian countries including India, the prevalence of smoking among women is low, where as smoking among men in high. These women live in environment, home, workplace, public places etc. where they are subjected to high rates of SHS exposure. But evidence from In India is little. Not many well conducted studies are available from India.

Kerala, the southernmost state of India, has health indicators in par with some of the developed countries. This state also has a high prevalence of smoking among men and women do not smoke. With such a high prevalence of smoking among men, pregnant women get exposed to passive smoke at home, workplace and public places. $^{9,10,11}$. The burden of various adverse pregnancy outcomes including stillbirth in women exposed to prenatal exposure to second hand smoke has not been studied in Kerala so far. Hence this study is designed to find out association of passive smoking with stillbirth. 
Most of the studies done in western literature and in developing countries where exposure of passive smoking and its effects is based on self-report. This study is done to quantify the effect of smoking by salivary cotinine.

\section{Objectives}

To study the association between SHS exposure during pregnancy and the risk of stillbirth.

\section{Methodology}

This study was conducted in the department of Obstetrics \& Gynaecology, Govt. Medical College, Trivandrum,a tertiary care teaching hospital. Study design is case -control study.

Cases: Antenatal women with sonologically confirmed stillbirth at or beyond 28 weeks gestation, willing to participate in the study were chosen as cases. All consecutive cases of antepartum stillbirths were recruited. For each case two cases of gestational age matched antenatal women with a live pregnancy registered in the same hospital were chosen as controls. case-control ratio 1:2. W.H.O criteria was used to define stillbirth, which is late fetal death at or beyond 28 wks of gestation was used. Stillbirth was diagnosed by ultrasound showing no evidence of fetal heart. A total of 64 cases and 128 controls were recruited for the study. Ethical clearance was obtained from the Institutional ethical committee.

After getting informed consent, data was collected using a pretested closed questionnaire. Details collected included demographic characteristics, age, socioeconomic status, religion, detailed obstetric history, menstrual history, BMI, Obstetric score, Booking status, Gestational Age, Weight gain in pregnancy, Maternal comorbidities such as Hypertension, Diabetes, Thyroid disease. Details of baby such as Sex, Birth weight, Congenital anomalies, Cord around neck were assessed after delivery

\section{Exposure Assessment}

Exposure to passive smoking was assessed by structured questionnaire. Number of smokers in family, type of product used to smoke and duration of smoke exposure by the antenatal women was noted. Questions also included exposure at work places, at movies and restaurants, at car and other places. Self-report of passive smoking was quantified by estimating salivary continine which is a byproduct of nicotine using ELISA test. Salivary continine assay has a high sensitivity and specificity and is a noninvasive test. After thorough mouth wash $3 \mathrm{ml}$ of saliva sample is collected from cases in a sterile bottle to measure the salivary cotinine level. The salivary sample is transported within 4 hours of collection in reverse cold chain box to Rajiv Gandhi Centre for biotechnology, Trivandrum for measuring the cotinine levels. Salivary cotine levels was measured at the molecular diagnostics division of Rajiv Gandhi Centre for biotechnology, Trivandrum. Biochemical analysis of salivary cotinine was done by using Enzyme Linked Immunosorbent Assay (ELISA) Kit imported from USA by Rajiv Gandhi Centre for biotechnology, Trivandrum. The Assay uses rabbit antibodies to cotinine and cotinine linked to horse radish peroxidase. The substrate used is tetramethylbenzidine. The ELISA Kit used has a sensitivity of $99.85 \%$ and a specificity of 93.37 $\%$.

\section{Data Analysis}

The data was entered into excel sheet. Analysis of data was done by statistical software(SSPS). Baseline characteristics were analysed using matched pair analysis. Odd's ratio and 95\% confidence interval was computed using matched pair analysis. Test of association was by Chisquare. Logistic regression was used to address confounding variables.

\section{Results}

The demographic and clinical characteristics of cases and controls were compared in Table1.There were 64 cases and 128 controls. The mean age of pregnant women recruited for study in both groups was $24.86+/-3.91$ years while that in the control group was $25.21+/-3.6$ years. Mean birth 
weight was 1.91 among cases and 2.26 among controls which was statistically significant ( t test2.695,p-0.0008). Preconception exposure to second hand smoke was reported by $75 \%$ of cases. In the control group $27.3 \%$ had exposure (OR 7.97, 95\% CI 4.01 -15.84)( p 0.001) (Table-2). They also reported exposure during pregnancy showing that exposure in the preconceptional period and during early gestation is a risk factor for stillbirth. Cigarette smoking was the commonest type of smoke reported by $60.9 \%$ of cases. Most of the cases reported exposure for three to five hours a week and none among controls were exposed for five hours a week. Also only among cases more than 2 members in the family smoked. Salivary cotinine was estimated for cases only. Mean cotinine level among subjects is 1.33 . Minimum cotinine level obtained in this study is 0.13 and maximum being 4.89 . (table-3) The seven times higher risk for stillbirth was unchanged even after control of confounders like socioeconomic status, gestational diabetes, and preeclampsia (table-4)

Table 1 Baseline characteristics

\begin{tabular}{|c|c|c|c|c|c|}
\hline \multirow[t]{2}{*}{ Study Variables } & & \multicolumn{2}{|c|}{ Case } & \multicolumn{2}{|c|}{ Control } \\
\hline & & $\mathbf{N}$ & $(\%)$ & $\mathbf{N}$ & $(\%)$ \\
\hline \multirow[t]{2}{*}{ Age } & $<30 \mathrm{yrs}$ & 24 & (37.5) & 30 & (23.4) \\
\hline & $>\quad 30 \mathrm{Yrs}$ & 40 & $(62.5)$ & 48 & (76.6) \\
\hline \multirow[t]{3}{*}{ Parity } & Primi & 23 & $(35.9)$ & 67 & $(52.3)$ \\
\hline & G2 & 20 & $(31.3)$ & 35 & $(27.3)$ \\
\hline & G3 & 21 & $(32.8)$ & 26 & \\
\hline \multirow{3}{*}{$\begin{array}{r}\text { Socio economic status } \\
\text { Middle }\end{array}$} & High & 1 & (1.6) & 1 & $(0.8)$ \\
\hline & & 40 & $(72.5)$ & 118 & $(80.2)$ \\
\hline & Low & 23 & $(35.9)$ & 19 & $(14.8)$ \\
\hline \multirow[t]{3}{*}{ BMI } & Underweight & 6 & $(9.35)$ & 12 & $(9.37)$ \\
\hline & Normal & 34 & $(53.12)$ & 91 & $(71.09)$ \\
\hline & Over weight & 24 & (37.5) & 25 & (19.53) \\
\hline
\end{tabular}

Table -2 Characteristics of second hand smoke exposure

\begin{tabular}{|c|c|c|c|c|c|c|c|}
\hline \multirow[t]{2}{*}{ Study Variables } & & \multicolumn{2}{|c|}{ Case } & \multicolumn{2}{|c|}{ Control } & \multirow[t]{2}{*}{$\mathbf{X}^{2}$} & \multirow[t]{2}{*}{$P$ value } \\
\hline & & $\mathbf{N}$ & $(\%)$ & $\mathbf{N}$ & $(\%)$ & & \\
\hline \multirow[t]{2}{*}{ Prenatal SHS exposure } & Yes & 48 & (25) & 35 & $(27.34)$ & 39.48 & 0.001 \\
\hline & No & 16 & $(25)$ & 93 & $(72.65)$ & & \\
\hline \multirow[t]{2}{*}{ Tobacco products } & cigarette & 39 & $(60.9)$ & 21 & $(16.4)$ & 46.2 & $<0.001$ \\
\hline & Beedi & 9 & $(15.6)$ & 14 & $(10.9)$ & & \\
\hline No of smoking & 0 & 15 & $(23.4)$ & 93 & $(72.7)$ & 44.6 & .000 \\
\hline \multirow[t]{2}{*}{ In the family } & 1 & 42 & $(67.2)$ & 34 & (26.6) & & \\
\hline & $\geq 2$ & 6 & & 0 & & & \\
\hline \multirow[t]{3}{*}{ Duration of exposure } & $<1 \mathrm{hr}$ & 5 & $(7.8)$ & 1 & $(0.8)$ & 69.2 & $<0.001$ \\
\hline & 3- $5 \mathrm{hr}$ & 42 & $(92)$ & 1 & $(0.8)$ & & \\
\hline & $>5 \mathrm{hr}$ & 1 & $(1.6 \%)$ & 0 & & & \\
\hline
\end{tabular}




\begin{tabular}{lc}
\multicolumn{2}{c}{ Table -3 Salivary cotinine level } \\
\hline & Salivary cotinine level \\
\hline $\mathrm{N}$ & 3.4 \\
Mean & 1.33 \\
Sd & 0.97 \\
Minimum & 0.13 \\
Maximum & 4.89 \\
Median & 1.27 \\
Q1 & 0.54 \\
Q3 & 1.71 \\
\hline
\end{tabular}

Table 4-logistic regression analysis

\begin{tabular}{lllll}
\hline Variables & OR & \multicolumn{1}{c}{$\mathbf{9 5 \%}$} & CI & P value \\
\hline Socio economic status & 1.97 & 0.824 & 4.2 & 0.075 \\
BMI & 2.89 & 1.27 & 6.55 & 0.011 \\
Gestational diabetes & 2.52 & 0.866 & 14.40 & 0.79 \\
SHS Exposure & 7.107 & 3.27 & 15.44 & 0.001 \\
\hline
\end{tabular}

\section{Discussion}

To our knowledge this is the first study done in the state of Kerala to assess the risk of second smoke exposure and stillbirth. Pregnant women who presented with sonologically confirmed evidence of still birth were recruited for this study and compared with pregnant women with live births. Case control ratio 1:2. We included only those cases at or beyond 28 weeks of gestation. Gestational age was determined from the last menstrual period and dating scan done in the first trimester of pregnancy. The history of passive smoking is elicited by a self administered questionnaire and self report of passive smoking was quantified by estimating salivary cotinine level which is a byproduct of nicotine using ELISA test. The key finding of our study is that those pregnant women exposed to second hand smoke prenatally and preconception ally had 7 times higher risk of antepartum stillbirth.

In the 64 subjects, 48 (i.e. $75 \%$ ) were exposed to passive smoking preconceptionally and during pregnancy giving a $\mathrm{p}$ value of $<0.001$ and an Odds ratio of 7.97, with a $95 \% \mathrm{CI} 4.01-15.84$, in both, showing a significant association. This association with second hand smoke exposure remained unchanged even after control of confounding variables. These findings were consistent with a meta-analysis done by Jo Leonardi-Bee et al ${ }^{13}$ assessing 19 studies on passive smoking and its effect on foetal outcome. Out of these 5 studies were analysed to study the risk of stillbirth on exposure to secondhand smoke. An overall assessment showed a 23\% increased risk association. Similar outcome was also seen in a previous study done by Miyake et al ${ }^{14}$ who studied the effects of environmental tobacco smoke on perinatal outcomes. Among the cases $60.9 \%$ were exposed to cigarette smoke and $15.6 \%$ to beedi smokers, and a relative increase in number of either per day increases the risk of developing stillbirth. In a study by Sreevidya et al, done in Mumbai, ${ }^{16}$ on pregnancy outcome was assessed after delivery. In that study second hand smoke exposure was based on self report. They found there was an association and stillbirth was higher among exposed group. The strength of our study is that the outcome was documented by ultrasound. The exposure status among cases was compared with gestational age matched live pregnancy. The self report of exposure was validated by estimating salivary cotinine. The 
seven times higher risk of stillbirth was unchanged even after controlling of confounders like age, BMI, gestational hypertension, and gestational diabetes.

\section{Policy implication}

The results of the study will be extremely useful for the public health policy makers to make statespecific intervention programmes for smoke cessation among males at home and in work places and thereby avoiding passive smoking in women and in their unborn babies. For health care programme managers, and health care providers to set up systems to identify second hand smoke exposure and thereby develop strategies to reduce preventable still birth due to SHS exposure.

\section{References}

1. WHO. International Classification of Diseases 10th revision (ICD-10). 2010. http://www.who.int/classifications/icd/ICD 10Volume2_en_2010.pdf?ua=1

2. WHO. World Health Organization. Implementing Smoke-free Environments. Rep Glob Tob Epidemic. 2009; WHO. Report on the Global Tobacco Epidemic. Implementing Smoke-free Environments. World Health Organization, 2009.Google Scholar

3. Centers for Disease Control and Prevention (US), U.S. Department of Health and Human Services. The Health Consequences of Involuntary Exposure to Tobacco Smoke: A Report of the Surgeon General. Publications and Reports of the Surgeon General. 2006. 727 p.

4. Wipfli H, Avila Tang E, Yuavan J, et al. Second hand smoke exposure among women and children : evidence from 31 countries. Am. J Public Health 2008;98:672-9

5. Grant SG; Quantitatively and Qualitatively similar effects of active and passive maternal tobacco use exposure: BMC Paediatrics 2005;5:20
6. The Partnership for Maternal, Newborn \& Child Health. Consultations on updating the Global Strategy for Women's, Children's and Adolescents' Health: round 2 -feedback on the zero draft of the Global Strategy. Geneva: The Partnership for Maternal, Newborn \& Child Health, 2015

7. Saving lives protecting futures-progress report on the global strategy for women's and children's health. 2015. http://www.everywoman everychild.org/global-strategy-2/gs2progress-report

8. The Lancet. The Lancet's Stillbirth Series. 2011. http://www. thelancet.com/series/stillbirth (accessed April 20, 2015).

9. K.T Shenoy, Richard Peto : Non communicable disease and tuberculosis burden in an urban cohort of 1,18772 subjects in Kerala, India

10. Ray JG, Urquia ML. Risk of stillbirth at extremes of birth weight between 20 to 41 weeks gestation. J Perinatol. 2012;32(11): 829-36.

11. Rakesh, P., Lalu, J. S., \& Leelamoni K. Prevalence of Exposure to Secondhand Smoke among Higher Secondary School Students in Ernakulam District, Kerala, Southern India. Journal of Pharmacy \& Bioallied Sciences, 9(1), Rakesh, P., Lalu, J. S., \& Leelamoni, K. (2017). Prevalence of Exposure to Sec. J Pharm Bioallied Sci. 2017;9(1):44-47.

12. Thankappan KR1 TC. Tobacco use \& social status in Kerala. Indian J Med Res. 2007;126(4):300-8.

13. Leonardi-Bee J, Britton J, Venn A. Secondhand Smoke and Adverse Fetal Outcomes in Nonsmoking Pregnant Women: A Meta-analysis. Pediatrics [Internet] 2011;127(4):734-41. Available from: http://pediatrics. aappublications. org/cgi/doi/10.1542/peds.2010-3041 
14. Miyake Y, Tanaka K, Arakawa M. Active and passive maternal smoking during pregnancy and birth outcomes: the Kyushu Okinawa Maternal and Child Health Study. BMC Pregnancy Childbirth [Internet]. 2013;13(1):157. Available from:

http://bmcpregnancychildbirth.biomedcent ral.com/articles/10.1186/1471-2393-13157

15. Dejmek J, Solansḱ y I, Podrazilová K, Šrám RJ. The exposure of nonsmoking and smoking mothers to environmental tobacco smoke during different gestational phases and fetal growth. Environ Health Perspect. 2002;110(6):601-6.

16. Subramoney S, Tursan d'Espaignet E, Chandra Gupta P. Higher risk of stillbirth among lower and middle income women who do not use tobacco, but live with smokers. Acta Obstet Gynecol Scand . 2010;89(4):572-7. 